\title{
Case Report \\ Severe Hypermagnesemia with Normal Renal Function Can Improve with Symptomatic Treatment
}

\author{
Yoshiaki Ishida $\mathbb{D}$ and Akihiko Tabuchi \\ Emergency and Critical Care Center, Anjo Kosei Hospital, 28 Higashihirokute, Anjo-cho, Anjo, Aichi 446-8602, Japan \\ Correspondence should be addressed to Yoshiaki Ishida; cogito_ergo_sum_yoshiaki@yahoo.co.jp
}

Received 29 March 2020; Revised 6 July 2020; Accepted 7 July 2020; Published 14 July 2020

Academic Editor: Aristomenis K. Exadaktylos

Copyright (c) 2020 Yoshiaki Ishida and Akihiko Tabuchi. This is an open access article distributed under the Creative Commons Attribution License, which permits unrestricted use, distribution, and reproduction in any medium, provided the original work is properly cited.

\begin{abstract}
Hypermagnesemia is a rare disorder and commonly occurs in patients with renal dysfunction. Supportive therapy for hypermagnesemia consists of administration of high-volume fluids, calcium preparation, diuretics, and, in severe cases, hemodialysis. Few reports have described severe hypermagnesemia patients with normal renal function who improved without hemodialysis. A 56-year-old woman presented with a history of constipation in spite of taking constipation medicine, including $\mathrm{MgO}$. She was brought to our emergency department due to vomiting and diffuse distension of the abdomen. Sudden vomiting, weakness, and lower level of consciousness occurred during examination. Her blood pressure dropped to $77 / 34 \mathrm{mmHg}$, and deep tendon reflexes of the limbs disappeared. Abdominal computed tomography showed bowel distension with wall edema, and biochemical testing showed serum $\mathrm{Mg}$ at $13.5 \mathrm{mg} / \mathrm{dl}$. She was diagnosed with severe hypermagnesemia associated with intestinal obstruction and administered intravenous loop diuretics and calcium preparation in addition to high volumes of normal saline. As the serum $\mathrm{Mg}$ level steadily declined, her level of consciousness returned to usual. This case suggests that severe hypermagnesemia can occur in patients with normal renal function and constipation under MgO. Severe hypermagnesemia with normal renal function can improve with symptomatic treatment without hemodialysis.
\end{abstract}

\section{Introduction}

Hypermagnesemia is rare and occurs most commonly in patients after ingestion of magnesium oxide $(\mathrm{MgO})$ [1]. Hypermagnesemia is caused mainly by decreased excretion from the kidney, increased absorption in the intestinal tract, or increased $\mathrm{Mg}$ intake [2]. Generally, hypermagnesemia occurs in elderly patients, especially with renal or bowel dysfunction $[3,4]$. Supportive therapy is the mainstay of the management of hypermagnesemia, involving high-volume fluids, diuretics, calcium preparation, and hemodialysis [5]. Notably, severe hypermagnesemia with renal failure and life-threatening symptoms usually requires hemodialysis $[3,6]$. However, few reports have described hypermagnesemia in patients with normal renal function $[1,5,7-9]$, and even fewer reports have described severe hypermagnesemia that improved with symptomatic treatment alone [7]. We report a severe hypermagnesemia patient with normal renal function, who was treated without hemodialysis.

\section{Case Presentation}

A 56-year-old woman presented with an 8-day history of constipation. She had been in a support facility for individuals with disabilities for many years due to Down's syndrome. She was brought to our emergency department (ED) due to vomiting and diffuse distension of the abdomen. She had a history of chronic constipation and had taken pharmacotherapies for constipation, including $\mathrm{MgO}$ at $1500 \mathrm{mg} /$ day. Physical examination of the abdomen revealed only nontender distension and tympanic sounds on percussion. Sudden vomiting, weakness, and reduced level of consciousness occurred during abdominal X-ray. While vital signs had been normal on arrival at our ED, her blood pressure dropped to $77 / 34 \mathrm{mmHg}$. She also showed a change to absence of deep tendon reflexes of the limbs. Electrocardiography showed a prolonged QT interval (Figure 1), abdominal X-ray showed accumulation of intestinal gas, and abdominal computed tomography showed bowel distension 


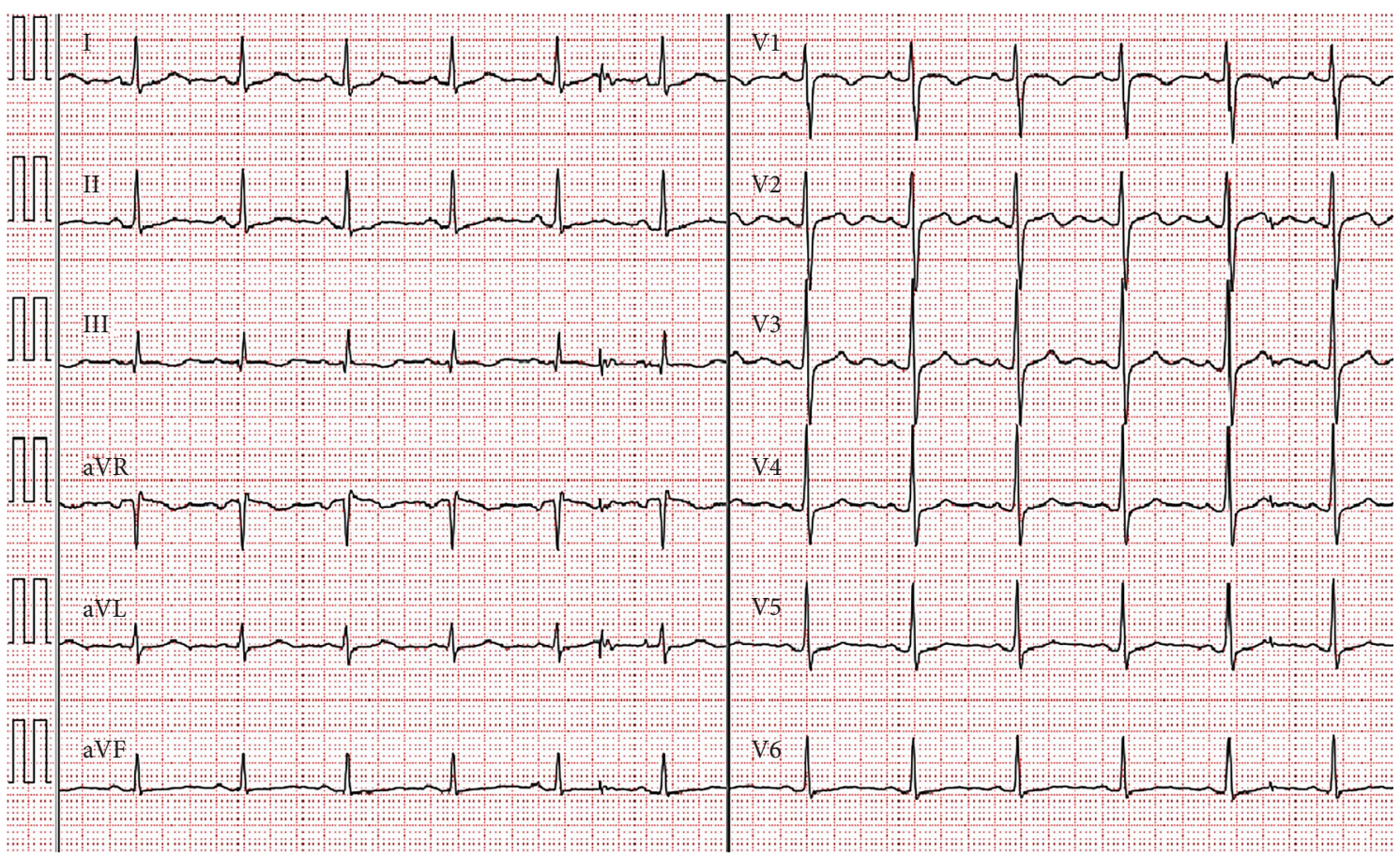

FIGURE 1: Electrocardiography on arrival in the emergency department demonstrating a prolonged QT interval (QT $442 \mathrm{~ms}$ and QTc $531 \mathrm{~ms}$ ).

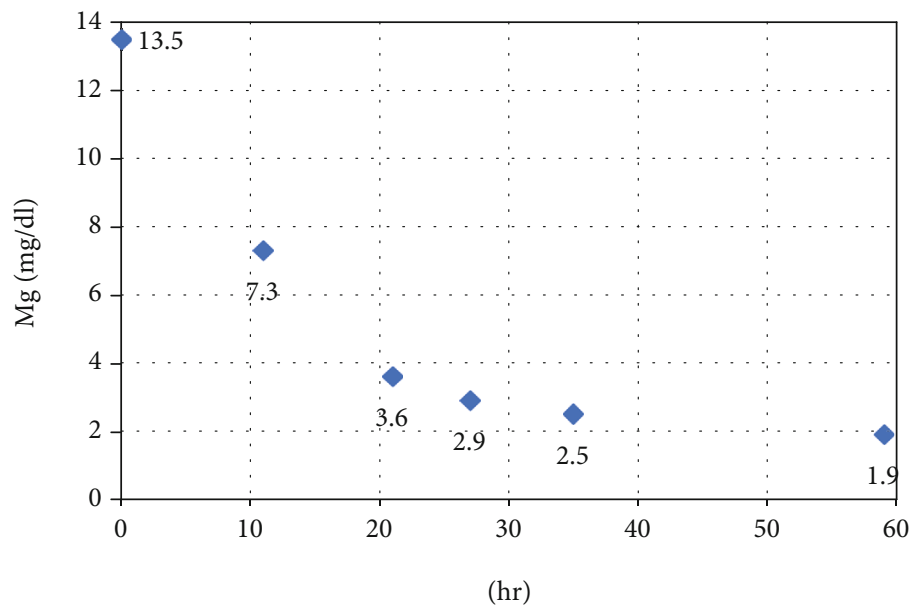

FIgURE 2: Changes in serum Mg values over time.

with wall edema. Biochemical testing showed the following: glucose, 135 (73-109) mg/dl; albumin, 3.0 (4.1-5.1) mg/dl; blood urea nitrogen, $18(8-20) \mathrm{mg} / \mathrm{dl}$; creatinine $(\mathrm{Cr}), 0.76$ $(0.46-0.79) \mathrm{mg} / \mathrm{dl}$; estimated glomerular filtration rate (eGFR), $61 \mathrm{ml} / \mathrm{min} / 1.73 \mathrm{~m}^{2}$; calcium, 9.2 (8.7-10.3) $\mathrm{mg} / \mathrm{dl}$; and $\mathrm{Mg}, 13.5$ (1.8-2.4) mg/dl. From the above, she was diagnosed with severe hypermagnesemia associated with intestinal obstruction. Although we consulted with her family to explore the option of emergency hemodialysis, they instead decided to continue symptomatic treatment. Intravenous loop diuretics and calcium preparation bolus were administered while infusing high volumes of normal saline. She subsequently showed good diuresis and a large amount of defecation the next day. Serum $\mathrm{Mg}$ level steadily declined, reaching $7.3 \mathrm{mg} / \mathrm{dl}$ after $11 \mathrm{~h}, 3.6 \mathrm{mg} / \mathrm{dl}$ after $21 \mathrm{~h}$, and
$2.9 \mathrm{mg} / \mathrm{dl}$ after $27 \mathrm{~h}$. By day 3, serum $\mathrm{Mg}$ level had almost normalized, at $2.5 \mathrm{mg} / \mathrm{dl}$ (Figure 2). As serum $\mathrm{Mg}$ level declined, her level of consciousness returned to usual. Eventually, she could walk unaided and was discharged on hospital day 15 with no sequelae.

\section{Discussion}

Hypermagnesemia is a relatively rare disorder and usually is caused by ingestion of $\mathrm{MgO}$ [1]. Hypermagnesemia is the least common electrolyte abnormality seen in the ED, accounting for only about $1 \%$ of electrolyte abnormality cases [10]. The normal range of serum $\mathrm{Mg}$ is $1.7-2.4 \mathrm{mg} / \mathrm{dl}$ [2], and symptoms start appearing when $\mathrm{Mg}$ concentrations exceed $5 \mathrm{mg} / \mathrm{dl}$. Symptoms include nausea/vomiting, cutaneous 
TABLE 1: Symptoms of hypermagnesemia associated with serum $\mathrm{Mg}$ levels.

\begin{tabular}{lc}
\hline Serum Mg level $(\mathrm{mg} / \mathrm{dl})$ & Clinical symptoms \\
\hline $1.7-2.4$ & Normal serum level \\
$5-8$ & $\begin{array}{c}\text { Nausea/vomiting, cutaneous flushing, } \\
\text { bradycardia, hypotension }\end{array}$ \\
$9-12$ & $\begin{array}{c}\text { Absent deep tendon reflexes, somnolence } \\
\text { Respiratory depression, paralysis, } \\
>15\end{array}$ \\
$>20$ & complete heart block \\
\hline
\end{tabular}

flushing, bradycardia, and hypotension [4]. Progression of hypermagnesemia results in the loss of deep tendon reflexes, somnolence, respiratory depression, paralysis, complete heart block, and even cardiac arrest [4] (Table 1). The present patient exhibited vomiting, hypotension, absence of deep tendon reflexes, and somnolence. She was diagnosed with severe hypermagnesemia because her serum Mg concentration $(13.5 \mathrm{mg} / \mathrm{dl})$ was at a life-threatening level.

We identified two important clinical issues. First, hypermagnesemia can occur in patients with bowel dysfunction even if renal function is normal and the $\mathrm{MgO}$ dose is within the upper limit. Generally, hypermagnesemia occurs in elderly patients, especially those with renal dysfunction or bowel dysfunction [2]. $\mathrm{Mg}$ reabsorption and regulation within the kidney principally occur at the epithelial cells of the thick ascending limb of Henle's loop [11]. Henle's loop has the capacity to completely reject $\mathrm{Mg}$ reabsorption under conditions of hypermagnesemia, and hence, a maximal renal excretion of more than $6 \mathrm{~g} /$ day (500 mEq/day) can occur [4]. Renal dysfunction thus leads to hypermagnesemia, and eGFR $<30 \mathrm{ml} / \mathrm{min} / 1.73 \mathrm{~m}^{2}$ is also associated with elevated risk of hypermagnesemia [7]. Mg regulation mainly occurs in the small intestine, particularly in the proximal portions [7], and its mechanism of regulation is mainly through passive diffusion [4]. Also, an elevation in $\mathrm{Mg}$ concentration is much more likely in the presence of gastrointestinal disorders (active gastric ulcer disease, gastritis, colitis) that can enhance $\mathrm{Mg}$ absorption [1]. Therefore, massive $\mathrm{Mg}$ ingestion may result in hypermagnesemia if the absorbed amount of $\mathrm{Mg}$ goes beyond the renal excretory capacity [4]. However, even if the dosage of oral $\mathrm{MgO}$ is within the normal range, hypermagnesemia still can occur depending on complications such as gastrointestinal disorders $[1,4,7,8,12-14]$. $\mathrm{MgO}$ is often used for chronic constipation, which promotes intestinal absorption of $\mathrm{Mg}$ due to long-term contact between the mucous membranes and $\mathrm{Mg}$. When serum Mg levels increase, movement of the intestinal tract is further decreased due to the neuromuscular blocking actions of $\mathrm{Mg}$ [7]. As a result, hypermagnesemia worsens. In our case, the patient had no history of renal disease and used $\mathrm{MgO}$ appropriately, but developed hypermagnesemia due to chronic constipation.

Our other key finding is that if renal function is normal, severe hypermagnesemia can improve with symptomatic treatment without hemodialysis. Two important supportive therapy principles for hypermagnesemia are removal of $\mathrm{Mg}$ from the blood and inhibition of $\mathrm{Mg}$ activity [12]. Removal of $\mathrm{Mg}$ from the blood can be achieved by high-volume normal saline, loop diuretics, and hemodialysis. Intravenous infusion of normal saline and loop diuretics enhances $\mathrm{Mg}$ excretion in patients with normal renal function [3]. In particular, loop diuretics inhibits $\mathrm{Mg}$ reabsorption in the thick ascending limb of Henle's loop [8]. Hemodialysis is necessary for patients with renal failure and life-threatening symptoms $[3,6]$. Inhibition of $\mathrm{Mg}$ activity requires administration of a calcium preparation to reverse cardiac arrhythmia, respiratory depression, and hypotension [3]. Many reports have described the treatment of hypermagnesemia with hemodialysis $[1-3,5-7,9,12,13]$. However, few reports have described hypermagnesemia in patients with normal renal function [1, 5, 7-9], and even fewer reports have described severe hypermagnesemia that improved with symptomatic treatment alone [7]. In our case, serum Mg levels in the patient normalized without hemodialysis. Intravenous administration of loop diuretics and calcium preparation while infusing high volumes of normal saline were sufficient to normalize $\mathrm{Mg}$ levels in our patient.

We have described a patient with normal renal function who developed severe hypermagnesemia because of chronic constipation and use of $\mathrm{MgO}$. In our case, hypermagnesemia with normal renal function can improve with symptomatic treatment without hemodialysis.

\section{Conflicts of Interest}

The authors declare that they have no competing interests.

\section{Acknowledgments}

The authors would like to thank FORTE (info@forte-science.co.jp) for performing an English language review.

\section{References}

[1] B. A. Clark and R. S. Brown, "Unsuspected morbid hypermagnesemia in elderly patients," American Journal of Nephrology, vol. 12, no. 5, pp. 336-343, 1992.

[2] T. Khairi, S. Amer, S. Spitalewitz, and L. Alasadi, "Severe symptomatic hypermagnesemia associated with over-thecounter laxatives in a patient with renal failure and sigmoid volvulus," Case Reports in Nephrology, vol. 2014, Article ID 560746, 2 pages, 2014.

[3] S. Onishi and S. Yoshino, "Cathartic-induced fatal hypermagnesemia in the elderly," Internal Medicine, vol. 45, no. 4, pp. 207-210, 2006.

[4] M. Kontani, A. Hara, S. Ohta, and T. Ikeda, "Hypermagnesemia induced by massive cathartic ingestion in an elderly woman without pre-existing renal dysfunction," Internal Medicine, vol. 44, no. 5, pp. 448-452, 2005.

[5] M. Shoaib Khan, S. Zahid, and M. Ishaq, "Fatal hypermagnesemia: an acute ingestion of Epsom salt in a patient with normal renal function," Caspian Journal of Internal Medicine, vol. 9, no. 4, pp. 413-415, 2018.

[6] A. Alaini, C. A. Roldan, K. Servilla, and E. S. Colombo, "Near death by milk of magnesia," BMJ Case Reports, vol. 2017, article bcr2016218260, 2017. 
[7] M. Nishikawa, N. Shimada, M. Kanzaki et al., "The characteristics of patients with hypermagnesemia who underwent emergency hemodialysis," Acute Medicine \& Surgery, vol. 5, no. 3, pp. 222-229, 2018.

[8] H. Yamaguchi, H. Shimada, K. Yoshita et al., "Severe hypermagnesemia induced by magnesium oxide ingestion: a case series," CEN Case Reports, vol. 8, no. 1, pp. 31-37, 2019.

[9] C. A. Weber and R. M. Santiago, "Hypermagnesemia. A potential complication during treatment of theophylline intoxication with oral activated charcoal and magnesium-containing cathartics," Chest, vol. 95, no. 1, pp. 56-59, 1989.

[10] A. K. Balc1, O. Koksal, A. Kose et al., "General characteristics of patients with electrolyte imbalance admitted to emergency department," World Journal of Emergency Medicine, vol. 4, no. 2, pp. 113-116, 2013.

[11] L. J. Dai and G. A. Quamme, "Intracellular Mg2+ and magnesium depletion in isolated renal thick ascending limb cells," The Journal of Clinical Investigation, vol. 88, no. 4, pp. 1255$1264,1991$.

[12] H. Tatsumi, Y. Masuda, H. Imaizumi et al., "A case of cardiopulmonary arrest caused by laxatives-induced hypermagnesemia in a patient with anorexia nervosa and chronic renal failure," Journal of Anesthesia, vol. 25, no. 6, pp. 935-938, 2011.

[13] Y. M. Weng, S. Y. Chen, H. C. Chen, J. H. Yu, and S. H. Wang, "Hypermagnesemia in a constipated female," The Journal of Emergency Medicine, vol. 44, no. 1, pp. e57-e60, 2013.

[14] T. S. Dharmarajan, B. Patel, and N. Varshneya, "Catharticinduced life threatening hypermagnesemia in a 90-year-old woman with apparent normal renal function," Journal of the American Geriatrics Society, vol. 47, no. 8, pp. 1039-1040, 1999. 